\title{
LIMITATIONS ON THE CONTROL OF SCHRÖDINGER EQUATIONS
}

\author{
Reinhard Illner $^{1},{\text { Horst } \text { Lange }^{2} \text { and Holger Teismann }}^{3}$
}

\begin{abstract}
We give the definitions of exact and approximate controllability for linear and nonlinear Schrödinger equations, review fundamental criteria for controllability and revisit a classical "No-go" result for evolution equations due to Ball, Marsden and Slemrod. In Section 2 we prove corresponding results on non-controllability for the linear Schrödinger equation and distributed additive control, and we show that the Hartree equation of quantum chemistry with bilinear control $(E(t) \cdot x) u$ is not controllable in finite or infinite time. Finally, in Section 3, we give criteria for additive controllability of linear Schrödinger equations, and we give a distributed additive controllability result for the nonlinear Schrödinger equation if the data are small.
\end{abstract}

Mathematics Subject Classification. 35Q40, 35Q55, 81Q99, 93B05.

Received September 30, 2004. Revised March 25 and May 3, 2005.

\section{INTRODUCTION}

We are concerned with controllability issues for linear and nonlinear Schrödinger and Hartree equations. The purpose of our article is threefold:

- to give a general overview on the concepts of exact and approximate controllability, and review (some of) the existing literature;

- to present a selection of results on non-controllability. This appears to be a rather generic scenario, and given that controls tend to be of particular type (consistent with physical or engineering limitations), it is not surprising that (exact) controllability seems to be the exception rather than the rule. These results are first steps towards the long-term goal of identifying the set of reachable states. They are also of interest in terms of the methodology developed to prove them.

We emphasize that the focus of this paper is exact (and approximate) controllability which is a rather ambitious control objective. Many of the equations considered in this paper which are found not to be exactly or approximately controllable may still be amenable to optimal control;

- to prove a few positive results, mostly for the linear case, and, in the nonlinear case, for the small data and small target case. Most of these results are new, though we use or adapt standard methodology.

\footnotetext{
Keywords and phrases. Schrödinger equations, exact and approximate control, quantum control.

1 Department of Mathematics and Statistics, University of Victoria, PO Box 3045, Victoria, B.C., V8W 3P4 Canada; rillner@math.uvic.ca

2 Mathematisches Institut, Universität Köln, Weyertal 86-90, 50931 Köln, Germany; lange@mathematik.uni-koeln.de

3 Department of Mathematics and Statistics, Acadia University, Wolfville, N.S., B4P 1R6 Canada;

hteisman@acadiau.ca
} 
We will distinguish two different types of control: additive control, where a control function, usually localized in time and space, appears as an inhomogeneity. The application of this type of control is for example meaningful in the nonlinear Schrödinger equation as it arises in nonlinear optics. The other type of control is known as bilinear control, and this is the control arising in quantum applications of Schrödinger equations. Here, the control function should be thought of as a (temporarily localized) modification of the external potential, and it will therefore multiply the wave function.

More detailed definitions of these types of controls are given in Section 1.1 below. There we also present the main types of Schrödinger equations to be considered, review well-known criteria for controllability, and list some basic classical results to be used later. In Section 1.3 we present a brief literature review.

Section 2 is devoted to results on non-controllability. First, in Section 2.1 we focus on linear Schrödinger equations with a general potential and give two different proofs for non-controllability with additive control (one based on a criterion from the book [45], the other a direct proof).

Section 2.2 contains our main results. Using as our point of departure an optimal control result for the nonlinear Hartree equation of quantum chemistry due to Cancès, LeBris, and Pilot [13], we give a proof (based on the Fourier transformation and on Hardy-Littlewood-Sobolev inequalities) that the standard bilinear control function $(E(t) \cdot x)$ does not allow exact or approximate control in finite time (cf. Th. 4). In addition, motivated by a general noncontrollability theorem for linear systems with bounded bilinear control due to Ball, Marsden, and Slemrod [3] (see Th. 2 below), we study the sets of reachable and non-reachable states for the Hartree equation for infinite control time. We prove that these sets are of first and second category, respectively ( $c f$. Th. 5). In particular, the set of non-reachable states turns out to be a dense subset of the state space, which makes our result analogous to the one by Ball, Marsden, and Slemrod. We conjecture that the set of reachable states is a non-dense subset of the state space, i.e., that the Hartree equation with control $(E(t) \cdot x)$ is not approximately controllable, even if the control time is allowed to become arbitrarily large.

In Section 2.3 we briefly turn to the controllability of Schrödinger equations with quadratic potentials such as the Gross-Pitaevskii equation with a quadratic trap. Utilizing a generalized WKB ansatz, we make the observation that the manifold of reachable states is actually finite-dimensional. Thus the system is very "far from" being controllable.

Section 3 contains what positive results we were able to prove. First, in Section 3.1, we consider a standard control problem for the linear Schrödinger equation with additive control. The control function there is of the type $g(t) h(x)$ with a fixed spatial profile $h(x)$. This form of the control arises when the full bilinear control problem is linearized. We are able to verify the approximate controllability criteria from [45] if the operator $A=-\Delta+V(x)$ possesses an orthonormal basis of eigenfunctions $\varphi_{n}$ in $L^{2}(\Omega)$, the corresponding eigenvalues satisfy a certain "gap condition", and the control profile $h(x)$ has non-vanishing inner products with every $\varphi_{n}$. Obviously, these conditions are rather strong ${ }^{1}$, and it would be desirable to relax them to be able to treat operators $A$ with continuous spectra etc. Having said that, we view our results as initial steps in developing a control theory for infinite-dimensional quantum systems - most of the known results deal with finite-dimensional quantum systems. The principal tool in the proofs of these results is the well-known Lemma of Ingham [21,44]. The proofs thus directly exploit the Schrödinger form of the equations. For real-valued control functions we need a real version of this lemma, for which we provide direct proof. Finally, in Section 3.2, we state a result on exact additive distributed control for the nonlinear Schrödinger equation with nonlinear term $-\alpha|u|^{2} u$, sufficiently small data and target states in $H^{1}$. This result (with a sketch of the proof) was presented in reference [20].

We begin by fixing the terminology.

\subsection{The terminology}

Let $x \in \mathbb{R}^{d}$, where $d$ is the dimension (usually $d=1,2$ or 3 ). The complex-valued function $u=u(t, x)$ is the state (or wave) function satisfying a (rescaled) generalized Schrödinger equation

$$
i u_{t}=A u-N(u)+B(g, u)
$$

\footnotetext{
${ }^{1}$ However, the important case of the anharmonic oscillator $A=-\frac{\mathrm{d}^{2}}{\mathrm{~d} x^{2}}+x^{2}+\lambda x^{4}$ is included.
} 
with initial value $u(0,)=.u_{0}(\cdot)$. Here $u_{t}$ denotes the time-derivative of $u, A=-\Delta+V(x)$ and $V$ is a potential function. $N$ is a nonlinear function (or functional) and $B$ is the control operator which depends on a usually real-valued function $g=g(t, x)$, and possibly on $u$ itself.

We distinguish the linear case

$$
N(u)=0
$$

and nonlinear cases, for example

$$
N(u)=-f\left(|u|^{2}\right) u .
$$

Of particular interest is the case $f(s)=s$, which produces the cubic nonlinearity.

As for the control operator, we consider (for example) the case where $B$ is independent of $u$, and

$$
B(g)=g(t) h(x)
$$

where $h$ is given and fixed; we call this additive control. Usually $h(x)=\chi_{\omega}(x)$ with some fixed open domain $\omega \subset \mathbb{R}^{d}$. This case is known as distributed control (as opposed to boundary control where $B$ is some boundary operator acting on the boundary of $\Omega$ ).

As mentioned earlier, this type of control is feasible for the Schrödinger equation as encountered in fiber optics, and the variable $t$ is then the longitudinal spatial variable.

Other controls of different physical relevance (namely in quantum physics) are

$$
B(g, u)=g(t) \tilde{B} u
$$

with a given linear operator $\tilde{B}$, and finally

$$
B(g, u)=\sum_{j=1}^{r} g_{j}(t) H_{j} u .
$$

We repeat that we will refer to (4) as additive control and to (5-6) as bilinear control (or quantum control). The $H_{j}$ are linear, usually Hamiltonian, operators.

Throughout this paper we assume that equation (1) admits strong or mild solutions on the given time interval $[0, T]$; for a given initial value $u_{0}, u(t)=u\left(t ; u_{0}, g\right)$ denotes the solution assuming $u_{0}$ initially and being driven by the control $g$. We think of $t \mapsto u(t)$ as a continuous function taking values in some complex Hilbert space (such as $H=L^{2}\left(\mathbb{R}^{d}\right), H=H^{1}\left(\mathbb{R}^{d}\right)$, or $H=H^{2}\left(\mathbb{R}^{d}\right)$ ). The control $g:[0, T] \rightarrow X$ takes values in another Hilbert space $X$.

Given an arbitrary "target" state $u_{T}$, the objective is to find a control $g$ such that $u\left(t ; u_{0}, g\right)=u_{T}$. The set of target states which are reachable from $u_{0}$ in time $T>0$ is given by

$$
R_{T}\left(u_{0}\right)=\left\{u\left(\tilde{T} ; u_{0}, g\right) \mid g \in L^{2}(0, \tilde{T} ; X), 0<\tilde{T} \leq T\right\}
$$

The set of all reachable states is defined as

$$
R\left(u_{0}\right):=\bigcup_{T>0} R_{T}\left(u_{0}\right)
$$

Definition 1. Equation (1) is called exactly controllable $[$ in time $T>0]$ if, for all $u_{0} \in H$,

$$
R\left(u_{0}\right)=H \quad\left[R_{T}\left(u_{0}\right)=H\right]
$$

Remark 1. Exact controllability in finite time is also known as strong complete controllability and the property $R\left(u_{0}\right)=H\left(\forall u_{0} \in H\right)$ is sometimes called complete controllability, see e.g. [14,18].

A slightly weaker notion of controllability is approximate controllability. 
Definition 2. We call equation (1) approximately controllable [in time $T>0$ ] if, for all $u_{0} \in H$,

$$
\overline{R\left(u_{0}\right)}=H \quad\left[\overline{R_{T}\left(u_{0}\right)}=H\right] .
$$

Remark 2. It is sometimes necessary to restrict the state space to a submanifold $S$ of $H$ to reflect certain a priori constraints on the control problem at hand. For instance, in many quantum applications the appropriate state space is given by

$$
S=\left\{h \in H \mid\|h\|_{L^{2}}=1\right\} .
$$

In these cases, the definitions above should be modified by replacing $H$ with $S$, where appropriate.

In the sequel we will always assume that the linear operator $A: D(A) \subset H \rightarrow H$ generates a continuous semi-group $S(t), t \geq 0$ on $H$.

We begin our discussion by listing a controllability criterion for additive control as presented in [45] for the problem

$$
\begin{aligned}
u_{t} & =A u+B g \\
u(0) & =u_{0},
\end{aligned}
$$

where the (u-independent) control operator $B$ is a bounded linear operator $X \rightarrow H$ and $g:[0, T] \rightarrow X$.

Theorem 1 (see, e.g., [45]). The problem (7) is exactly controllable at $T>0$ if and only if the bounded linear operator $B^{*} S(t)^{*}: H \rightarrow L^{2}(0, T ; X)$ is positive definite, i.e., if there is a $C>0$ such that for all $h \in H$

$$
\int_{0}^{T}\left\|B^{*} S(t)^{*} h\right\|^{2} \mathrm{~d} t \geq C\|h\|^{2} .
$$

Furthermore, (7) is approximately controllable at $T>0$ if and only if the identity $B^{*} S(t)^{*} h=0$, for almost all $t \in[0, T]$, implies that $h \equiv 0$.

In the bilinear control case $(B(g, u)=g(t) \tilde{B} u)$ there is a strong negative result due to Ball, Marsden and Slemrod [3].

Theorem 2 (Ball, Marsden and Slemrod, 1982). Let $\operatorname{dim} H=\infty$, and suppose that $\tilde{B}: X \rightarrow H$ is linear and bounded. Then, for any $u_{0} \in H$, the set $R\left(u_{0}\right)$ is contained in a countable union of compact subsets of $H$, and the set of non-reachable elements $H \backslash R\left(u_{0}\right)$ is dense in $H$.

\section{Remark 3.}

(i) It should be noted that this "No-go" theorem does not exclude the possibility of the system being approximately controllable. In fact, Ball, Marsden and Slemrod do present conditions under which the set of reachable states $R\left(u_{0}\right)$ will be dense (for suitable choices of the initial state $u_{0}$ ). Their conditions are applicable to certain (classical) bilinear control problems associate with the wave and rod equations subject to various boundary conditions. For details, see [3], Sections 5 and 6 .

(ii) Turinici adapted the theorem to the quantum situation (linear Schrödinger equation with bounded bilinear control). His version [42,43] reads: Let $H=H^{2}\left(\mathbb{R}^{d}\right)$ and $u_{0} \in S:=\left\{h \in H \mid\|h\|_{L^{2}}=1\right\}$. Then the set of reachable states is contained in a countable sets of (relatively) compact subsets of $S$. In particular, the set of non-reachable states is a (relatively) dense subset of $S$.

(iii) It should be pointed out that the controllability of partial differential equations may depend sensitively on the regularity of the states and controls. Non-controllability may be the result of a poor choice of the state space w.r.t. to the space of controls; controllability may be recovered by working in a different (higher-regularity) state space. This phenomenon occurs in two recent papers by Beauchard and Coron $[8,9]$. The bilinear control problem considered there falls in the scope of the noncontrollabilty result by Turinici if the state space is chosen to be $H^{2}$. However, the authors show that the problem is locally 
controllable if the state space is altered to $H^{7}$. (We are grateful to one of the referees for pointing these papers out to us.)

(iv) There is a another fundamental result on the infinite-dimensional quantum control problem due to Huang, Tarn and Clark $[14,18]$ who consider the linear case $(N(u)=0$ in $(1))$ with a control operator of the form (6). Using ideas from geometric control theory, the controllability criterion is formulated in terms of various Lie algebras generated by the Hamiltonians involved. One consequence of the "HTC Theorem," as it is known, can be formulated as follows [18]. If the Lie algebra generated by the operators $A, H_{1}, \ldots, H_{r}$ is finite-dimensional and admits an analytic domain ${ }^{2} D \subset H$, then equation (1) is not analytically controllable on $S$; i.e., $R\left(u_{0}\right) \neq S \cap D\left(u_{0} \in S \cap D\right)$.

These theorems set the stage for our discussion of controllability issues for Schrödinger equations. Before we present our observations and results, we list a few of the standard examples and give a brief overview over the literature.

\subsection{Examples}

(i) The interaction of a quantum system with its environment can be modelled by a Schrödinger equation of the form ${ }^{3}$

$$
i u_{t}=H_{0} u+H_{\text {int }}(t) u,
$$

where $H_{0}$ is the (time-independent) Hamiltonian of the unperturbed quantum system - typically, $H_{0}=-\Delta+V(x)-$ and the (time-dependent) Hamiltonian $H_{\text {int }}(t)$ describes the interaction. In control applications the interaction operator $H_{e x t}(t)$ is given by the R.H.S. of (6), where the functions $g_{j}(t)$ represent classical fields by means of which the quantum system is manipulated. The interaction Hamiltonian in the dipole approximation is given by the operator $E(t) \cdot x$; i.e., $g_{j}(t)=E_{j}(t)$ and $H_{j}=x_{j}$, where $E(t)=\left(E_{1}(t), E_{2}(t), E_{3}(t)\right)$ denotes the (classical) electric field of a laser, say ${ }^{4}$. For more details, see $[10,37]$ and the literature therein. (For alternate choices of the operators $H_{j}$ see also $[14,24]$.) A large amount of literature exists on the controllability of finite-dimensional quantum systems. (A number of important references may be found in [39], for example.) Specific infinitedimensional quantum systems are treated in [35] and [24] which deal with the control of the quantumharmonic oscillator and the hydrogen atom, respectively.

(ii) The particle-particle interaction in many-body quantum systems is often modelled by a nonlinear, self-consistent, potential, which leads to the Hartree equation; the corresponding control problem with dipole term for these models takes the form

$$
i u_{t}=-\Delta u+V(x) u+\alpha\left(|u|^{2} * \frac{1}{|x|}\right) u+(E(t) \cdot x) u
$$

$(\alpha \in \mathbb{R}$ ). In quantum-chemistry applications (control of molecular processes) $V(x)$ is typically given by the Coulomb potential $\frac{Z}{|x|}$; in the theory of semi-conductors $V(x)$ may be a periodic or confining potential.

(iii) The Gross-Pitaevskii equation

$$
i u_{t}=-\Delta u+V(x) u+\alpha|u|^{2} u
$$

describes a Bose-Einstein condensate in a mean field approximation. Here $V(x)$ is the magnetic trap holding the condensate; typically $V(x)$ is a quadratic function of $x$. In this paper we consider the case where the control is again given by the dipole term $E(t) \cdot x$ (see, e.g., [17]). However, other parameters,

\footnotetext{
2 This means that $D$ is a dense subset of $H$ which is invariant w.r.t. the operators $A, H_{1}, \ldots, H_{r}$ and on which the solutions to the Schrödinger equation (1) can be expressed globally in exponential form [14,18].

${ }^{3}$ All physical constants have been set to 1 .

${ }^{4}$ In practice the unbounded operator $x$ is often replaced with a bounded one.
} 
such as trap frequency and scattering length, can also be influenced and tuned in the laboratory. It is therefore interesting to study the effects of these additional control "knobs" as well [1].

(iv) It is well known that nonlinear Schrödinger equations have important applications in nonlinear optics. The controls in these systems are provided by holding or pump beams; the appropriate mathematical form is therefore given by the additive control term. The "fixed-profile" form (4) considered in Section 3.1 arises when only the strength of the holding beam is varied.

\subsection{A brief literature review}

As opposed to the massive available body of knowledge on controllability of finite and infinite-dimensional classical systems, only a limited amount of literature on exact and optimal control of Schrödinger equations is available.

An excellent introduction to control questions for Schrödinger equations is [46]. Results on distributed and/or boundary control for linear Schrödinger equations are the subjects of references [15,25, 27-29,33,34,36]; local exact controllability for the linear Schrödinger equation with bilinear control is shown in [8,9]. A small-data result on distributed exact control for the nonlinear Schrödinger equation was presented in [20] (for completeness, this result is listed in Sect. 3 below). References $[5,7,13]$ present optimal control results for the (nonlinear) Hartree equation and bilinear controls. Our Theorem 4 complements these results.

For comprehensive surveys on the control of quantum systems and the relevant literature we refer the reader to the two recent monographs $[10,37]$ as well as $[30,42]$. Computational aspects of Quantum Chemistry are the subject of [31]. The articles $[14,18]$ contain the result on bilinear control of infinite-dimensional quantum systems described in Remark 3 (iii) above.

Many introductory texts on control theory are available; we only mention [45] which also contains results on infinite-dimensional systems (see Th. 1 above). Standard references on control theory for partial differential equations include e.g. $[4,26,32,38]$. Finally, the general "No-go" Theorems by Ball, Marsden, Slemrod (Th. 2) and Turinici (Rem. 3 (ii)) have already been cited.

\section{Results ON NON-CONTROLLABILITY}

\subsection{Linear Schrödinger equations}

We prove the impossibility of exact controllability for a whole class of linear Schrödinger equations with additive control.

We recall the controllability criteria as presented in the introduction [45] for the problem

$$
\begin{aligned}
u_{t} & =A u+B g \\
u(0) & =u_{0}
\end{aligned}
$$

where $B: X \rightarrow H$ is a linear operator acting on the controls $g:[0, T] \rightarrow X$.

By Theorem 1 equation (8) is exactly controllable at $T>0$ if and only if the bounded linear operator $B^{*} S(t)^{*}: H \rightarrow L^{2}(0, T ; X)$ is positive definite, i.e., there is a $C>0$ such that for all $\varphi \in H$

$$
\int_{0}^{T}\left\|B^{*} S(t)^{*} \varphi\right\|^{2} \mathrm{~d} t \geq C\|\varphi\|^{2} .
$$

We consider Schrödinger equations of the type

$$
\begin{aligned}
i u_{t} & =-\Delta u+V(x) u+\chi_{\omega}(x) g(t, x) \\
u(0, x) & =u_{0}(x)
\end{aligned}
$$

in the internally distributed additive control form, with open subsets $\omega \subset \Omega \subset \mathbb{R}^{d}, H=L^{2}(\Omega)$ and a real potential $V ; \Omega$ may be $\mathbb{R}^{d}$ itself, but $\omega$ should be bounded. 
In the sequel we state two conditions each one of which implies the impossibility of exact controllability for (9).

(i) $-i(-\Delta+V)$ generates a group (the appropriate Schrödinger group) $S(t)_{t \in \mathbb{R}}$ such that an $L^{1}-L^{\infty}$ estimate of the type

applies; or,

$$
\|S(t)\|_{L^{1} \rightarrow L^{\infty}} \leq C|t|^{-d / 2}(\forall t \neq 0)
$$

(ii) $-\Delta+V$ has a discrete spectrum $\left\{\lambda_{n}\right\}_{n \in \mathbb{N}}$ of eigenvalues with eigenfunctions $\left\{\varphi_{n}\right\}$ such that $\left\{\varphi_{n}\right\}$ is an orthonormal basis of $H=L^{2}(\Omega)$, and

$$
\int_{\omega}\left|\varphi_{n}\right|^{2} \mathrm{~d} x=o(1) \text { as } n \rightarrow \infty .
$$

We remark that the first condition is satisfied in the free case $(V=0)$ or if the real potential $V$ is such that the multiplication operator defined by multiplication with $(1+|x|)^{1 / 2} V(x)$ maps $H^{s}\left(\mathbb{R}^{d}\right)$ into itself $(s>0)$, and $\hat{V} \in L^{1}\left(\mathbb{R}^{d}\right)$ (if $\Omega=\mathbb{R}^{d}$ ) (see [22]). The second criterion is, for example, satisfied for the harmonic oscillator $V(x)=x^{2}$ on $\Omega=\mathbb{R}^{d}$, as discussed in [20].

Theorem 3. If either of the above conditions is satisfied, then (9) is not exactly controllable.

Proof. Suppose the first condition applies. For simplicity we only discuss the case $\Omega=\mathbb{R}^{d}$ and $V \equiv 0$ (the "free" case; the general case is quite similar).

Let $\psi_{\epsilon}$ be a set of regularizing functions on $\mathbb{R}^{d}$ such that

$$
\begin{gathered}
\psi_{\epsilon} \in C_{0}^{\infty}, \psi_{\epsilon} \geq 0 \\
\int \psi_{\epsilon} \mathrm{d} x=1, \quad \operatorname{supp} \psi_{\epsilon}=\{x ;|x| \leq \epsilon\},
\end{gathered}
$$

and

$$
\int\left|\psi_{\epsilon}\right|^{2} \mathrm{~d} x=C \epsilon^{-d}
$$

Let $S(t)$ denote the free Schrödinger group, given explicitly by

$$
(S(t) \varphi)(x)=(4 \pi i t)^{-d / 2} \int \exp \left(i \frac{|x-y|^{2}}{4 t}\right) \varphi(y) \mathrm{d} y
$$

(defined for $\varphi \in L^{1}\left(\mathbb{R}^{d}\right)$ ). Then, set $\varphi_{\epsilon}:=S(2 T) \psi_{\epsilon}$. We observe that

$$
\left\|\varphi_{\epsilon}\right\|_{L^{2}}^{2}=\left\|S(2 T) \psi_{\epsilon}\right\|_{L^{2}}^{2}=\left\|\psi_{\epsilon}\right\|_{L^{2}}^{2}=C \epsilon^{-d} .
$$

If we now assume exact controllability of (9), it would follow from Theorem 1 that there is a constant $C_{T}>0$ such that

$$
\begin{aligned}
C \epsilon^{-d} & =\left\|\varphi_{\epsilon}\right\|_{L^{2}}^{2} \leq C_{T} \int_{0}^{T} \int\left|\chi_{\omega} S(-t) \varphi_{\epsilon}\right|^{2} \mathrm{~d} x \mathrm{~d} t \\
& =C_{T} \int_{0}^{T} \int_{\omega} 1 \cdot\left|S(2 T-t) \psi_{\epsilon}\right|^{2} \mathrm{~d} x \mathrm{~d} t \leq \tilde{C}_{T}|\omega| \int_{0}^{T} \frac{\mathrm{d} t}{(2 T-t)^{d}}\left(\int \psi_{\epsilon} \mathrm{d} y\right)^{2} \\
& \leq C_{0}(T, \omega, d)<\infty,
\end{aligned}
$$

and this gives a contradiction for small $\epsilon$. 
We turn to the second criterion above. In this case we have for any $\varphi \in L^{2}(\Omega)$

$$
(S(t) \varphi)(x)=\sum_{k \in \mathbb{N}} \mathrm{e}^{-i \lambda_{k} t}\left(\varphi, \varphi_{k}\right)_{L^{2}(\Omega)} \varphi_{k}(x) .
$$

As $\left\|\varphi_{n}\right\|_{L^{2}(\Omega)}=1$ and $S(-t) \varphi_{n}=\mathrm{e}^{i \lambda_{n} t} \varphi_{n}$, we have

$$
\int_{0}^{T} \int_{\Omega}\left|\chi_{\omega} S(-t) \varphi_{n}\right|^{2} \mathrm{~d} x \mathrm{~d} t=T \int_{\omega}\left|\varphi_{n}\right|^{2} \mathrm{~d} x=o(1)
$$

for $n \rightarrow \infty$. Therefore the operator $B^{*} S(-t)$ cannot be positive definite from $L^{2}\left(0, T ; L^{2}(\Omega)\right)$ to $L^{2}(\Omega)$, i.e., (9) is not exactly controllable.

Remark 4. The theorem may also be proved in a more direct way without reference to Theorem 1, using the notion of observable controllability: define

$$
\mathcal{O}_{T, C}\left(u_{0}\right):=\left\{u_{T}=u\left(T ; u_{0}, g\right) \mid\|g\|_{L^{2}\left(0, T ; L^{2}\left(\mathbb{R}^{d}\right)\right)} \leq C\left(\left\|u_{0}\right\|_{L^{2}}+\left\|u_{T}\right\|_{\left.L^{2}\right)}\right\} ;\right.
$$

i.e.,

$$
R_{T}\left(u_{0}\right)=\bigcup_{C>0} \mathcal{O}_{T, C}\left(u_{0}\right) .
$$

We will show that for all $u_{0} \in L^{2}(\Omega)$ and any $C>0$ there is a target state $u_{T}$ such that $u_{T} \notin \mathcal{O}_{T, C}\left(u_{0}\right)$, which will obviously imply that the equation is not exactly controllable. To show this, fix $u_{0}$ and $C>0$ and assume that $\varphi_{\epsilon} \in \mathcal{O}_{T, C}\left(u_{0}\right)$ for all $\epsilon>0$. Then there exist controls $g_{\epsilon} \in L^{2}\left(0, T ; L^{2}\left(\mathbb{R}^{d}\right)\right) \subset L^{1}\left(0, T ; L^{2}\left(\mathbb{R}^{d}\right)\right)$ such that

$$
\left\|g_{\epsilon}\right\|_{L^{2}\left(0, T ; L^{2}(\omega)\right)} \leq C\left\{\left\|u_{0}\right\|_{L^{2}}+\left\|\varphi_{\epsilon}\right\|_{L^{2}}\right\}
$$

and $\varphi_{\epsilon}=u\left(T ; u_{0}, g_{\epsilon}\right)$. Hence

and

$$
\varphi_{\epsilon}=S(T) u_{0}-i \int_{0}^{T} S(T-t)\left(\chi_{\omega} g_{\epsilon}\right) \mathrm{d} t
$$

$$
C \epsilon^{-d}=\left(S(T) u_{0}, S(2 T) \psi_{\epsilon}\right)_{L^{2}\left(\mathbb{R}^{d}\right)}-\int_{0}^{T} \int_{\omega} g_{\epsilon}(x, t) \overline{S(T+t) \psi_{\epsilon}} \mathrm{d} x,
$$

which, using (10), implies

$$
\begin{aligned}
C \epsilon^{-d} & \leq\left\|u_{0}\right\|_{L^{2}}\left\|\psi_{\epsilon}\right\|_{L^{2}}+C \int_{0}^{T} \frac{\mathrm{d} t}{(T-t)^{d / 2}} \int_{\omega}\left|g_{\epsilon}(t, x)\right| \mathrm{d} x \\
& \leq C\left\|u_{0}\right\|_{L^{2}} \epsilon^{-d / 2}+C(T, d, \omega)\left\|g_{\epsilon}\right\|_{L^{2}(\omega)} \\
& \leq C\left\|u_{0}\right\|_{L^{2}} \epsilon^{-d / 2}+\tilde{C}\left\{\left\|u_{0}\right\|+C \epsilon^{-d / 2}\right\} .
\end{aligned}
$$

By multiplying with $\epsilon^{-d / 2}$ one arrives at a contradiction for small enough $\epsilon$.

In the case of assumption (11) we argue in a similar manner: let $u_{0}$, and $C$ be fixed and assume that $\varphi_{n} \in \mathcal{O}_{T, C}\left(u_{0}\right)$ for all $n$; denote by $g_{n} \in L^{2}\left(0, T ; L^{2}(\Omega)\right)$ the appropriate control. Then

$$
\varphi_{n}=S(T) u_{0}-i \int_{0}^{T} \sum_{k \in \mathbb{N}} \mathrm{e}^{-i \lambda_{k}(T-t)}\left(\chi_{\omega} g_{n}(t, \cdot), \varphi_{k}\right) \varphi_{k} \mathrm{~d} t
$$

and therefore

$$
1=\left(S(T) u_{0}, \varphi_{n}\right)_{L^{2}(\Omega)}-i \int_{0}^{T} \mathrm{e}^{-i \lambda_{k}(T-t)}\left(g_{n}(t, \cdot), \varphi_{n}\right)_{L^{2}(\omega)} \mathrm{d} t .
$$


This leads to an estimate

$$
1 \leq\left|\left(S(T) u_{0}, \varphi_{n}\right)_{L^{2}(\Omega)}\right|+C\left\{\left\|u_{0}\right\|+1\right\}\left\{\int_{\omega}\left|\varphi_{n}\right|^{2} \mathrm{~d} x\right\}^{1 / 2} .
$$

This gives a contradiction for large $n$ because $\left(\varphi_{n}\right)$ converges weakly to 0 , and, by assumption, we know that $\int_{\omega}\left|\varphi_{n}\right|^{2} \mathrm{~d} x \rightarrow 0$ as $n \rightarrow \infty$.

\subsection{The Hartree equation}

In this section we prove two noncontrollability results (Ths. 4 and 5) for the nonlinear Hartree equation of quantum chemistry (see Eq. (16) below). The first theorem deals with control in finite time and is motivated by an optimal control result due to Cancès, LeBris, and Pilot [13] who proved that optimal control problem

$$
\inf _{E \in L^{2}\left(0, T ; \mathbb{R}^{3}\right)} J(E)=\inf _{E \in L^{2}\left(0, T ; \mathbb{R}^{3}\right)}\left(\left\|u\left(T ; u_{0}, E\right)-u_{T}\right\|_{L^{2}\left(\mathbb{R}^{3}\right)}^{2}+\|E\|_{L^{2}(0, T)}^{2}\right)
$$

has a solution $E \in L^{2}\left(0, T ; \mathbb{R}^{3}\right)$ for every $u_{0} \in H^{2,1}\left(\mathbb{R}^{3}\right):=\left\{f \in H^{2}\left(\mathbb{R}^{3}\right) \mid\left(1+|x|^{2}\right)^{1 / 2} f \in L^{2}\left(\mathbb{R}^{3}\right)\right\}$ and $u_{T} \in L^{2}\left(\mathbb{R}^{3}\right)$.

In view of this result, a natural question to ask is whether the distance $\left\|u\left(T ; u_{0}, E\right)-u_{T}\right\|_{L^{2}}$ between the attainable states $u\left(T ; u_{0}, E\right)$ and the target state $u_{T}$ can be made small (possibly at the cost of large controls $E(t))^{5}$. Theorem 4 and Remark 5 (ii) show that this is impossible in general.

In the second theorem we consider the infinite-time control problem for (16). Here the question is whether the results on the noncontrollability of linear systems with bounded bilinear control by Ball, Marsden, Slemrod and Turinici (see Th. 2 and Rem. 3 (ii)) have an analogue for the nonlinear Hartree equation with the unbounded dipole control term. In Theorem 5 we show that this is indeed the case.

The equation under consideration has the normalized form

$$
i u_{t}=-\Delta u-\frac{1}{|x|} u+\left(|u|^{2} * \frac{1}{|x|}\right) u+(E(t) \cdot x) u
$$

$\left(x \in \mathbb{R}^{3}, t \in[0, T]\right), u(0, x)=u_{0}(x)$. Here the real-valued field vector $E(t)=\left(E_{1}(t), E_{2}(t), E_{3}(t)\right)^{T}$ is the control function. It can be seen from $[6,12,13]$ that equation (16) is globally well-posed in $H:=H^{2}\left(\mathbb{R}^{3}\right)$, with

$$
u \in C([0, T], H) \cap C^{1}\left([0, T], L^{2,-1}\left(\mathbb{R}^{3}\right)\right),
$$

where

$$
L^{2,-1}\left(\mathbb{R}^{3}\right):=\left\{f \in L_{l o c}^{2}\left(\mathbb{R}^{3}\right) \mid\left(1+|x|^{2}\right)^{-1 / 2} f \in L^{2}\left(\mathbb{R}^{3}\right)\right\}
$$

To formulate our results, we recall the definitions of the sets of reachable states in finite time, $R_{T}\left(u_{0}\right)$ $=\left\{u\left(\tilde{T} ; u_{0}, g\right) \mid g \in L^{2}(0, \tilde{T} ; X), 0<\tilde{T} \leq T\right\}$, and infinite time, $R\left(u_{0}\right):=\bigcup_{T>0} R_{T}\left(u_{0}\right)$, and we define the sets

$$
\begin{aligned}
S & :=\left\{h \in H \mid\|h\|_{L^{2}}=1\right\} \\
N R_{T}\left(u_{0}\right) & :=\mathcal{C} R_{T}\left(u_{0}\right) \\
U_{T}\left(u_{0}\right) & :=\left\{h \in S\left|\sup _{0<\tilde{T} \leq T} \sup _{E \in L^{2}\left(0, \tilde{T} ; \mathbb{R}^{3}\right)}\right|\left\langle u\left(\tilde{T} ; u_{0}, E\right), h\right\rangle_{L^{2}} \mid<1\right\} \\
\Sigma_{T}\left(u_{0}\right) & :=\left\{h \in S\left|\sup _{0<\tilde{T} \leq T} \sup _{E \in L^{2}\left(0, \tilde{T} ; \mathbb{R}^{3}\right)}\right|\left\langle u\left(\tilde{T} ; u_{0}, E\right), h\right\rangle_{L^{2}} \mid=1\right\} .
\end{aligned}
$$

\footnotetext{
5 One could introduce a parameter $\left(\alpha>0\right.$, say) in front of the "fluence" term $\|E\|_{L^{2}(0, T)}^{2}\left(i . e\right.$. replace $\|E\|_{L^{2}(0, T)}^{2}$ in the functional $J$ with $\left.\alpha\|E\|_{L^{2}(0, T)}^{2}\right)$ and try to "solve" the exact control problem by taking the limit $\alpha \rightarrow 0$.
} 
Note that

$$
U_{T}\left(u_{0}\right)=\mathcal{C} \Sigma_{T} \subset N R_{T}\left(u_{0}\right) \text { and } \overline{R_{T}\left(u_{0}\right)} \subset \Sigma_{T}
$$

We regard $S$ as a complete metric space with the the metric $d(g, h)=\|g-h\|_{H^{2}}$. The subsets of $S$ are topological spaces w.r.t. the relative topology; $\mathcal{C}$ and $\overline{(.)}$ denote the (set-theoretic) complement and the topological closure in $S$, respectively.

Lemma 1. Let $u_{0} \in S$ and $T>0$. Then the set $U_{T}\left(u_{0}\right)$ is a dense and open subset of $S$.

The following theorem is now a direct consequence of (17).

Theorem 4. Let $u_{0} \in S$ and $T>0$. Then $\overline{R_{T}\left(u_{0}\right)} \neq S$; i.e., the equation (16) is not approximately controllable in time $T$. Moreover, the set of non-reachable states, $N R_{T}\left(u_{0}\right)$, contains a dense and open subset of $S$.

Before proving the lemma, we make a few comments.

\section{Remark 5.}

(i) Although the observation that equation (16) is not approximately controllable in finite time may come as no surprise, given the special form of the control term $(E(t) \cdot x) u$, the theorem is interesting for several reasons: a) the control term arises naturally in the theory of light-matter interaction (dipole approximation $)^{6}$ b) equation (16) is optimally controllable as mentioned above - the theorem therefore says that the states $u\left(T ; u_{0}, E\right)$ can in general not be made arbitrarily close to the target state $u_{T}$ even if the "fluence" $\|E\|_{L^{2}(0, T)}^{2}$ of the control $E$ is allowed to become arbitrarily large. (See also Rem. (ii) below.); c) the dependence of the "degree of controllability" on the external potential is highly nontrivial. For instance, if the Coulomb potential is replaced by the harmonic oscillator potential, the set of reachable states turns out to be finite-dimensional ( $c f$. Sect. 2.3), which, however, it is clearly not in the Coulomb case; $d$ ) the method for proving the theorem is of independent interest.

(ii) As mentioned in the introduction to this section, our main motivation for the theorem has been to interpret the optimal control result by Cancés, LeBris, and Pilot cited above in the context of exact control. In this regard, we have that

$$
\left\{u_{T} \in S \mid \inf _{0<\tilde{T} \leq T} \inf _{E \in L^{2}(0, \tilde{T})}\left\|u\left(\tilde{T} ; u_{0}, E\right)-u_{T}\right\|_{L^{2}} \geq \alpha\right\} \neq \emptyset
$$

for all $\alpha \in(0, \sqrt{2})$, which illustrates the "gap" between exact and optimal controllability for the Hartree equation.

(iii) The theorem also holds and is of independent interest in the linear case (hydrogen atom).

(iv) We will make use of the Hardy-Littlewood-Sobolev inequality in the following form: Let $0<\alpha<d$, and for $f \in L^{p}\left(\mathbb{R}^{d}\right)$ define the Riesz potential $R_{\alpha}(f)(x)$ by

$$
R_{\alpha}(f)(x):=\int \frac{f(y)}{|x-y|^{\alpha}} \mathrm{d} y .
$$

Then there exists a constant $C=C_{\alpha, d}>0$ such that for all exponents $p, q$ with $1 \leq p<q<\infty, \quad \frac{1}{q}$ $=\frac{1}{p}+\frac{\alpha}{d}-1$,and all $f \in L^{p}\left(\mathbb{R}^{d}\right)$ the estimate

$$
\left\|R_{\alpha}(f)\right\|_{L^{q}} \leq C\|f\|_{L^{p}}
$$

holds. We will use (18) for the case $d=3, q=2, \alpha=2$ and $p=6 / 5$.

\footnotetext{
${ }^{6}$ In quantum-chemistry computations the unbounded operator $x$ may be replaced with a bounded one by, for instance, applying a cut-off at infinity.
} 
(v) We will use the following formula for the Fourier transform of $\frac{1}{|x|}$ (see, for example, [41]):

$$
\widehat{\left(\frac{1}{|x|}\right)}(\xi)=\lambda_{d}\left(\frac{1}{|\xi|}\right)^{d-1}
$$

with $\lambda_{d}=\pi^{\frac{1-d}{2}} \Gamma\left(\frac{\alpha-1}{2}\right)$.

(vi) As mentioned in Remark 3 (iii), varying the state space may change the controllability properties dramatically. Here, however, it is not the regularity of the states which is the obstacle to controllability. Rather, the decay properties at infinity seem to be more important. Our proof, in its present form, does not extend to the space $H^{2,1}\left(\mathbb{R}^{3}\right)$, which is the natural solution space for equation (16).

Proof of Lemma 1.

Step 1. Construction of special elements of $U_{T}\left(u_{0}\right)$. Consider the sequence $\left(g_{n}\right)_{n \in \mathbb{N}} \subset S$ of functions $g_{n}$ defined by

$$
g_{n}(x):=n^{-3 / 2} g\left(\frac{x}{n}\right)
$$

where $g \in C^{\infty}\left(\mathbb{R}^{3}\right)$ is an arbitrary function such that $\|g\|_{L^{2}}=1$. Then

$$
\left\|g_{n}\right\|_{L^{2}}=\|g\|_{L^{2}}=1 \quad \text { and } \quad\left\|\hat{g}_{n}\right\|_{L^{6 / 5}}=n^{-1}\|\hat{g}\|_{L^{6 / 5}}
$$

for all $n \in \mathbb{N}$.

Let $u=u\left(. ; u_{0}, E\right) \in C(0, T ; H)$ be the unique solution of

$$
\left\{\begin{aligned}
i u_{t} & =-\Delta u-\frac{1}{|x|} u+\left(|u|^{2} * \frac{1}{|x|}\right) u+(E(t) \cdot x) u \\
u(0, x) & =u_{0}(x)
\end{aligned}\right\} .
$$

We claim that $g_{n} \in U_{T}$ for $n$ large enough; more precisely, we will show that

$$
\left|\left\langle u\left(\tilde{T} ; u_{0}, E\right), g_{n}\right\rangle_{L^{2}}\right| \longrightarrow 0 \quad(n \rightarrow \infty) \quad \text { uniformly in } \tilde{T} \in(0, T] \text { and } E \in L^{2}(0, \tilde{T}) .
$$

We start by noting that the $L^{2}$ norm of $u$ is conserved,

$$
\|u(t, .)\|_{L^{2}}=\left\|u_{0}\right\|_{L^{2}}=\left\|u\left(T ; u_{0}, E\right)\right\|_{L^{2}} \quad(0 \leq t \leq T) .
$$

Moreover, taking the Fourier transform of (20) and using

$$
[(E(t) \cdot x) u]^{\curlywedge}(\xi)=i E(t) \cdot \nabla_{\xi} \hat{u} \quad \text { and } \quad \widehat{f \cdot g}=(2 \pi)^{-d / 2} \hat{f} * \hat{g}
$$

results in the transport equation

$$
\hat{u}_{t}-E(t) \cdot \nabla_{\xi} \hat{u}=-i \xi^{2} \hat{u}+i c_{0}\left(\frac{1}{|\xi|^{2}} * \hat{u}\right)-i c_{1}(W(u) * \hat{u}),
$$

where $W(u):=\widehat{V(u)}, V(u):=\frac{1}{|x|} *|u|^{2}, c_{1}:=(2 \pi)^{-3 / 2}, c_{0}:=\lambda_{3} c_{1}$ (the constant $\lambda_{3}$ is defined in Remark 5 (v) above). The homogeneous equation associated with equation (23) (i.e. Eq. (23) without the last two terms on the right-hand side) has the solution

$$
\hat{u}_{0}(\xi+H(t)) \cdot \exp \left[-i \int_{0}^{t}(\xi+H(t)-H(s))^{2} \mathrm{~d} s\right]
$$


where $H(t):=\int_{0}^{t} E(\tau) \mathrm{d} \tau$. Using the Duhamel Principle, a representation of the solution of (23) can be obtained:

$$
\begin{aligned}
\hat{u}(t, \xi)= & \hat{u}_{0}(\xi+H(t)) \mathrm{e}^{-i \int_{0}^{t}(\xi+H(t)-H(s))^{2} \mathrm{~d} s} \\
& +i \int_{0}^{t}\left(c_{0}\left(\frac{1}{|\xi|^{2}} * \hat{u}\right)-c_{1}(W(u) * \hat{u})\right)(s, \xi) \cdot \mathrm{e}^{-i \int_{s}^{t}(\xi+H(t)-H(\sigma))^{2} \mathrm{~d} \sigma} \mathrm{d} s .
\end{aligned}
$$

Thus

$$
\begin{aligned}
\hat{u}(T, \xi)= & \hat{u}_{0}(\xi+H(T)) \mathrm{e}^{-i \int_{0}^{T}(\xi+H(T)-H(s))^{2} \mathrm{~d} s} \\
& +i \int_{0}^{T}\left(c_{0}\left(\frac{1}{|\xi|^{2}} * \hat{u}\right)-c_{1}(W(u) * \hat{u})\right)(s, \xi) \cdot \mathrm{e}^{-i \int_{s}^{T}(\xi+H(T)-H(\sigma))^{2} \mathrm{~d} \sigma} \mathrm{d} s \\
=: & I_{1}+I_{2}+I_{3},
\end{aligned}
$$

where

$$
\begin{aligned}
& I_{1}:=\hat{u}_{0}(\xi+H(T)) \mathrm{e}^{-i \int_{0}^{T}(\xi+H(T)-H(s))^{2} \mathrm{~d} s} \\
& I_{2}:=i c_{0} \int_{0}^{T}\left(\frac{1}{|\xi|^{2}} * \hat{u}\right)(s, \xi) \cdot \mathrm{e}^{-i \int_{s}^{T}(\xi+H(T)-H(\sigma))^{2} \mathrm{~d} \sigma} \mathrm{d} s \\
& I_{3}:=-i c_{1} \int_{0}^{T}(W(u) * \hat{u})(s, \xi) \cdot \mathrm{e}^{-i \int_{s}^{T}(\xi+H(T)-H(\sigma))^{2} \mathrm{~d} \sigma} \mathrm{d} s .
\end{aligned}
$$

Now we multiply equation (26) by $\overline{\hat{g}_{n}}$, and integrate over $\mathbb{R}^{3}$.

To estimate the first term in

$$
\left\langle u\left(T ; u_{0}, E\right), g_{n}\right\rangle_{L^{2}}=\left\langle I_{1}, \hat{g}_{n}\right\rangle_{L^{2}}+\left\langle I_{2}, \hat{g}_{n}\right\rangle_{L^{2}}+\left\langle I_{3}, \hat{g}_{n}\right\rangle_{L^{2}},
$$

we first assume that $\hat{u}_{0} \in L^{6}\left(\mathbb{R}^{3}\right)$, and get by Hölder's inequality,

$$
\left|\left\langle I_{1}, \hat{g}_{n}\right\rangle_{L^{2}}\right| \leq\left\|I_{1}\right\|_{L^{6}}\left\|\hat{g}_{n}\right\|_{L^{6 / 5}} \leq\left\|\hat{u}_{0}\right\|_{L^{6}}\left\|\hat{g}_{n}\right\|_{L^{6 / 5}} \leq n^{-1}\left\|\hat{u}_{0}\right\|_{L^{6}}\|\hat{g}\|_{L^{6 / 5}} \longrightarrow 0 .
$$

Now the the general case can be dealt with by a simple density argument.

Using the Remark 5 (v) and (vi) as well as the conservation law (22), we estimate

$$
\begin{aligned}
\left|\left\langle I_{2}, \hat{g}_{n}\right\rangle_{L^{2}}\right| & =c_{0} \mid \int_{0}^{T} \int_{\mathbb{R}^{3}} \int_{\mathbb{R}^{3}} \frac{\hat{u}(s, \eta) \mathrm{e}^{-i \int_{s}^{T}(\xi+H(T)-H(\sigma))^{2} \mathrm{~d} \sigma} \overline{\hat{g}_{n}(\xi)} \mathrm{d} \eta \mathrm{d} \xi \mathrm{d} s \mid}{|\xi-\eta|^{2}} \\
& \leq c_{0} \int_{0}^{T} \int_{\mathbb{R}^{3}}\left(\int_{\mathbb{R}^{3}} \frac{\left|\hat{g}_{n}(\xi)\right|}{|\xi-\eta|^{2}} \mathrm{~d} \xi\right)|\hat{u}(s, \eta)| \mathrm{d} \eta \mathrm{d} s \\
& =c_{0} \int_{0}^{T} \int_{\mathbb{R}^{3}} R_{2}\left(\left|\hat{g}_{n}\right|\right)(\eta) \cdot|\hat{u}(s, \eta)| \mathrm{d} \eta \mathrm{d} s \\
& \leq c_{0}\left\|R_{2}\left(\left|\hat{g}_{n}\right|\right)\right\|_{L^{2}} \int_{0}^{T}\|u(s, .)\|_{L^{2}} \mathrm{~d} s=C\left\|R_{2}\left(\left|\hat{g}_{n}\right|\right)\right\|_{L^{2}} \\
& \leq C^{\prime}\left\|\hat{g}_{n}\right\|_{L^{6 / 5}} \leq C^{\prime} n^{-1}\|\hat{g}\|_{L^{6 / 5}} \longrightarrow 0 .
\end{aligned}
$$

(The constant $C^{\prime}$ depends linearly on $T$.) The term $\left|\left\langle I_{3}, \hat{g}_{n}\right\rangle_{L^{2}}\right|$ can be treated in a similar manner:

$$
\left|\left\langle I_{3}, \hat{g}_{n}\right\rangle_{L^{2}}\right| \leq C^{\prime \prime}\left(\int_{\mathbb{R}^{3}}|u(s, y)|^{2} \mathrm{~d} y\right) \cdot\left|\left\langle\hat{u}(s, .), R_{2}\left(\hat{g}_{n}\right)\right\rangle_{L^{2}}\right| \leq C^{\prime \prime} n^{-1}\|\hat{g}\|_{L^{6 / 5}}
$$


where we have used $\left|\widehat{|u|^{2}}\right| \leq\|u\|_{L^{2}}^{2}$. This completes the proof of (21).

Step 2. $U_{T}\left(u_{0}\right)$ is a dense subset of $S$. Let $u_{T} \in S$ and $\delta>0$. We will show that the state

$$
\tilde{u}_{T, n}:=\frac{u_{T}+\varepsilon g_{n}}{\left\|u_{T}+\varepsilon g_{n}\right\|_{L^{2}}} \in S
$$

satisfies

$$
\left\|\tilde{u}_{T, n}-u_{T}\right\|_{H^{2}}<\delta \quad \text { and } \quad \tilde{u}_{T, n} \in U_{T}\left(u_{0}\right)
$$

if $\varepsilon>0$ and $n \in \mathbb{N}$ are suitably chosen numbers. To this end, we first observe that

$$
\left\|g_{n}\right\|_{H^{2}} \leq C
$$

where $C=C\left(\|g\|_{H^{2}}\right)$ is a constant that only depends on the $H^{2}$-norm of $g$. We choose $\varepsilon>0$ such that

$$
\frac{\varepsilon}{\sqrt{1+\varepsilon^{2} / 2}}\left(\left\|u_{T}\right\|_{H^{2}}+C\right)<\delta .
$$

Since

$$
\left\|u_{T}+\varepsilon g_{n}\right\|_{L^{2}}^{2} \geq 1+\varepsilon^{2}-2\left|\left\langle u_{T}, g_{n}\right\rangle_{L^{2}}\right|
$$

and $\left|\left\langle u_{T}, g_{n}\right\rangle_{L^{2}}\right| \rightarrow 0$, we may choose a number $n_{1} \in \mathbb{N}$ such that

$$
\left\|u_{T}+\varepsilon g_{n}\right\|_{L^{2}}^{2} \geq 1+\varepsilon^{2} / 2
$$

for all $n \geq n_{1}$. Now

$$
\begin{aligned}
\left\|\tilde{u}_{T, n}-u_{T}\right\|_{H^{2}} & =\frac{1}{\left\|u_{T}+\varepsilon g_{n}\right\|_{L^{2}}} \cdot\left\|u_{T}\left(\left\|u_{T}+\varepsilon g_{n}\right\|_{L^{2}}-1\right)-\varepsilon g_{n}\right\|_{H^{2}} \\
& \leq \frac{1}{\sqrt{1+\varepsilon^{2} / 2}} \cdot\left(\left|\left\|u_{T}+\varepsilon g_{n}\right\|_{L^{2}}-\left\|u_{T}\right\|_{L^{2}}\right| \cdot\left\|u_{T}\right\|_{H^{2}}+\varepsilon\left\|g_{n}\right\|_{H^{2}}\right) \\
& \leq \frac{\varepsilon}{\sqrt{1+\varepsilon^{2} / 2}}\left(\left\|u_{T}\right\|_{H^{2}}+\left\|g_{n}\right\|_{H^{2}}\right)<\delta
\end{aligned}
$$

(for all $n \geq n_{1}$ ) and

$$
\begin{aligned}
\left|\left\langle u\left(T ; u_{0}, E\right), \tilde{u}_{T, n}\right\rangle_{L^{2}}\right|= & \frac{1}{\left\|u_{T}+\varepsilon g_{n}\right\|_{L^{2}}}\left|\left\langle u\left(T ; u_{0}, E\right), u_{T}\right\rangle_{L^{2}}+\varepsilon\left\langle u\left(T ; u_{0}, E\right), g_{n}\right\rangle_{L^{2}}\right| \\
\leq & \frac{1}{\sqrt{1+\varepsilon^{2} / 2}}\left(1+\varepsilon\left|\left\langle u\left(T ; u_{0}, E\right), g_{n}\right\rangle_{L^{2}}\right|\right) \\
& \longrightarrow \frac{1}{\sqrt{1+\varepsilon^{2} / 2}}<1,
\end{aligned}
$$

since $\left|\left\langle u\left(T ; u_{0}, E\right), g_{n}\right\rangle_{L^{2}}\right| \rightarrow 0$ (see (21)). This completes the proof of (30).

Step 3. $U_{T}\left(u_{0}\right)$ is an open subset of $S$. Define the map $\phi_{T}: S \rightarrow \mathbb{R}$,

$$
\phi_{T}(h):=\sup _{0<\tilde{T} \leq T} \sup _{E \in L^{2}\left(0, \tilde{T} ; \mathbb{R}^{3}\right)}\left|\left\langle u\left(T ; u_{0}, E\right), h\right\rangle_{L^{2}}\right| .
$$

This map is clearly continuous, since it is the restriction to $S$ of the sublinear map $\tilde{\phi}_{T}: H \rightarrow \mathbb{R}, \tilde{\phi}_{T}(h)$ $:=\sup _{0<\tilde{T} \leq T} \sup _{E \in L^{2}\left(0, \tilde{T} ; \mathbb{R}^{3}\right)}\left|\left\langle u\left(T ; u_{0}, E\right), h\right\rangle_{L^{2}}\right|$ which satisfies

$$
\tilde{\phi}_{T}(h) \leq 1 \quad(\forall h \in H) .
$$


Thus, the set

$$
U_{T}=\left\{h \in S \mid \phi_{T}(h)<1\right\}
$$

is open by the continuity of $\phi_{T}$, which completes the proof of Lemma 1 .

We now turn to the infinite-control-time result. (For information on first- and secondary-category sets etc., we refer the reader to $[2]$, e.g.)

Theorem 5. Let $u_{0} \in S$. Then

(i) The set of reachable states, $R\left(u_{0}\right)$, is of first category ("meagre") in $S$.

(ii) The set of non-reachable states, $N R\left(u_{0}\right)$, is of second category ("fat") in $S$. In particular, $N R\left(u_{0}\right)$ is dense in $S$.

\section{Remark 6.}

(i) It can be shown (using the convergence results in [13], Sect. 3.3) that the sets $R_{T, C}\left(u_{0}\right):=\left\{u\left(T ; u_{0}, E\right) \mid\right.$ $\left.E \in L^{2}(0, T),\|E\|_{L^{2}(0, T)} \leq C\right\}$ are compact subsets of $L^{2}\left(\mathbb{R}^{3}\right)$. The density of $N R\left(u_{0}\right)$ in the topology of $L^{2}\left(\mathbb{R}^{3}\right)$ (which is obviously weaker than the $H^{2}\left(\mathbb{R}^{3}\right)$-topology) may therefore also be shown using this compactness property as the set of reachable states,

$$
R\left(u_{0}\right)=\bigcup_{n, N \in \mathbb{N}} R_{n, N}\left(u_{0}\right),
$$

is a countable union of compact sets and thus has a $\left(L^{2}\right.$-)dense complement $N R\left(u_{0}\right)$.

(ii) It seems likely that equation (16) is not approximately controllable; i.e. that $\overline{R\left(u_{0}\right)} \neq S$, but at the present time we do not have a complete proof for this conjecture.

(iii) As before, we would also like to generalize the theorem to include the topology of $H^{2,1}\left(\mathbb{R}^{3}\right)$.

Proof of Theorem 5. As before, for each $T>0$, we consider the map $\phi_{T}: S \rightarrow \mathbb{R}$, defined in (31) above. In addition to the set $U_{T}=\left\{h \in S \mid \phi_{T}(h)<1\right\}$ we will also need the set

$$
\Sigma_{T}:=\left\{h \in S \mid \phi_{T}(h)=1\right\} .
$$

Evidently, $U_{T}$ is open and $\Sigma_{T}$ is closed by the continuity of $\phi_{T}$.

(i) Clearly,

$$
R\left(u_{0}\right)=\bigcup_{n \in \mathbb{N}} R_{n}\left(u_{0}\right),
$$

so it is sufficient to show that $R_{T}\left(u_{0}\right)$ is nowhere dense for each $T>0$. First, note that

$$
\overline{R_{T}\left(u_{0}\right)} \subset \Sigma_{T} .
$$

It therefore suffices to show that

$$
\left(\Sigma_{T}\right)^{\circ}=\emptyset .
$$

To see this, let $h \in \Sigma_{T}$. Then, thanks to Lemma 1, we have that $h \in \overline{U_{T}}$ which implies that any open neighbourhood of $h$ contains elements of $U_{T}=\mathcal{C} \Sigma_{T}$. This shows that the set $\Sigma_{T}$ does not contain any inner points; so its interior $\left(\Sigma_{T}\right)^{\circ}$ is empty.

(ii) The sets $U_{T}$ are open and dense and therefore of second category ("fat"). Thus

$$
U:=\bigcap_{n \in \mathbb{N}} U_{n}
$$

is a second-category set as a countable intersection of second-category sets. The assertion now follows from $U \subset N R\left(u_{0}\right)$. 


\subsection{Schrödinger equations with quadratic potentials}

In this section we briefly consider the control problem for nonlinear Schrödinger equations with quadratic potentials:

$$
i u_{t}=-\Delta u+\left(x^{T} \Omega x\right) u+(E(t) \cdot x) u+f\left(|u|^{2}\right) u
$$

$\left(x \in \mathbb{R}^{3}, t \in[0, T]\right), u(0, x)=u_{0}(x)$. Here $E$ has the same meaning as before; $\Omega$ denotes a symmetric $(d \times d)$ matrix; the nonlinearity $f\left(|u|^{2}\right)$ may be local or nonlocal. Equation (33) thus encompasses the Gross-Pitaevskii $\left(f\left(|u|^{2}\right)=a|u|^{2}\right)$ and Hartree $\left(f\left(|u|^{2}\right)=V(u)=\frac{1}{|x|} *|u|^{2}\right)$ equations with quadratic exterior potentials.

The critical observation is that the control term can be removed from the equation by a (generalized) WKBtype ansatz

$$
u(t, x)=\mathrm{e}^{i S(t, x)} \psi(t, x-\bar{x}(t)),
$$

where the function $S$ is appropriately chosen and $\bar{x}(t)$ denotes the classical trajectory associated with the classical Hamiltonian

$$
\bar{H}(p, x)=\frac{1}{2} p^{2}+x^{T} \Omega x+E(t) \cdot x .
$$

Transformations of this kind are known for the linear quantum-mechanical oscillator [19,23] and have recently been utilized in the nonlinear case (for time-independent "Stark potentials" $E \cdot x$ ) as well [11]. The equation for $\psi$ takes the form

$$
i \psi_{t}=-\Delta \psi+\left(x^{T} \Omega x\right) \psi+f\left(|\psi|^{2}\right) \psi,
$$

which is independent of the control $E$. We therefore draw the following conclusion.

Theorem 6. Let $u_{0} \in H^{2,2}\left(\mathbb{R}^{d}\right)$ and $T>0$. Then, for any control $E \in L^{2}\left(0, T ; \mathbb{R}^{d}\right)$, the solution $u=u\left(. ; u_{0}, E\right)$ of equation (33) is given by (34), where $\psi$ satisfies (35) and $\psi(0, x)=u_{0}(x)$. In particular, the manifold of reachable states is given by

$$
R_{T}\left(u_{0}\right)=\left\{\mathrm{e}^{i \lambda} \mathrm{e}^{i \pi \cdot(x-\xi)} \psi(T, x-\xi) \mid \lambda \in \mathbb{R} ; \pi, \xi \in \mathbb{R}^{d}\right\}
$$

and is hence finite-dimensional.

This obviously means that the degree to which the system can be controlled is very limited, as the state $\psi(T,$. can be pre-computed independently of the control field $E$. As a result, all that can be affected by the application of the control field is - up to a phase factor - a shift of the state $\psi(T,$.$) . This limitation on the controllabil-$ ity of (33) is particularly striking if the initial state $u_{0}$ is chosen to be a nonlinear eigenstate for (33); i.e., $-\Delta u_{0}+\left(x^{T} \Omega x\right) u_{0}+f\left(\left|u_{0}\right|^{2}\right) u_{0}=\mu u_{0}(\mu \in \mathbb{R})$. In this case, the density of the controlled state, $|u(T, x)|^{2}=$ $\left|u_{0}(x-\bar{x}(T))\right|^{2}$, remains unchanged (up to the shift $\bar{x}(T)$ ) by the application of the homogeneous control field $E$.

It is also interesting to compare Theorem 6 (in the linear case $f \equiv 0$ ) to the results in $[8,9]$, which show that (local) controllability is recovered if the quadratic potential is replaced with an "infinite" confining potential ("particle in a box"). This illustrates the subtle dependence of the controllability properties on the external potential.

\section{Controllability Results}

In this section we present two controllability results for Schrödinger equations.

\subsection{Additive control for the linear Schrödinger equation}

We begin with the problem

$$
\left\{\begin{aligned}
i u_{t} & =A u+g(t) h(x) \quad(x \in \Omega, \quad t \in[0, T]) \\
u(0) & =u_{0}
\end{aligned}\right\}
$$


where $A$ is a linear differential operator such as $-\Delta+V(x), h$ is a given function, and $\Omega=\mathbb{R}^{d}$ or $\Omega \subset \mathbb{R}^{d}$ bounded and open subset of $\mathbb{R}^{d}$ (in the last case equation (36) is to be understood subject to boundary conditions, such as $\left.\left.u\right|_{(0, T) \times \partial \Omega}=0\right)$.

Problems of the form (36) (where we distinguish between the real case $\Im(g)=0$ and the complex case) are of interest since they appear as linearizations of bilinear control situations ${ }^{7}$. Their solutions may therefore be an important step in obtaining local controllability results for bilinear control problems. While we need strong assumptions on the spectrum of the Hamiltonian (discreteness, gap condition), which we would eventually like to be able to relax, our conditions do include important examples such as the (one-dimensional) anharmonic oscillator $-\frac{d^{2}}{\mathrm{~d} x^{2}}+x^{2}+\lambda x^{4}(\lambda \in \mathbb{R})$, whose eigenvalues are known to behave like $\left(n+\frac{1}{2}\right)^{4 / 3} \lambda^{1 / 3}$ [40]. The proofs are based on the following result by Ingham $(c f .[21,44])$ on nonharmonic Fourier series, which seems to restrict the method to Schrödinger equations.

Lemma 2. Let $f \in L^{2}(0, T ; \mathbb{C})$ be given by

$$
f(t)=\sum_{k=1}^{\infty} c_{k} \mathrm{e}^{i \lambda_{k} t}
$$

where $c_{k} \in \mathbb{C}$ such that $\sum_{k=1}^{\infty}\left|c_{k}\right|^{2}<\infty$ and $\left(\lambda_{k}\right) \subset \mathbb{R}$.

If $\left(\lambda_{n}\right)$ is a separated sequence of real numbers satisfying the gap condition

$$
\lambda_{n+1}-\lambda_{n} \geq \gamma>\frac{2 \pi}{T} \quad(n \in Z)
$$

then the system $\left\{\mathrm{e}^{i \lambda_{n} \tau}\right\}$ is a Riesz-Fischer sequence in $L^{2}(0, T)$; i.e., there exist constants $C_{T}$ and $\tilde{C}_{T}$ such that

$$
C_{T} \sum_{k=1}^{\infty}\left|c_{k}\right|^{2} \leq \int_{0}^{T}|f(t)|^{2} \mathrm{~d} t \leq \tilde{C}_{T} \sum_{k=1}^{\infty}\left|c_{k}\right|^{2}
$$

Remark 7. The inequalities in (39) imply that the collection of functions $\left(\mathrm{e}^{i \lambda_{k} t}\right)_{k \in \mathbb{N}}$ form a Riesz basis of $L^{2}(0, T ; \mathbb{C})$. Moreover, any function $f$ of the form $(37)$ is uniquely determined by its coefficients if the gap condition (38) and the condition $T>2 \pi / \gamma$ are satisfied.

We also need a version of the uniqueness assertion of the previous lemma for real numbers for which we provide a direct proof.

Lemma 3. Let $\left(\lambda_{n}\right) \subset \mathbb{R} \backslash\{0\}$ be a sequence of non-zero numbers that satisfies the gap condition (38). Then there exists a number $T_{0}>3 \pi / \gamma$ such that for every $T \geq T_{0},\left(\alpha_{n}\right),\left(\beta_{n}\right) \subset \mathbb{R}$ s.t. $\sum_{n=1}^{\infty}\left(\left|\alpha_{n}\right|+\left|\beta_{n}\right|\right)<\infty$, the real non-harmonic Fourier series

$$
f(t)=\sum_{n=1}^{\infty}\left\{\alpha_{n} \sin \left(\lambda_{n} t\right)+\beta_{n} \cos \left(\lambda_{n} t\right)\right\} \quad(t \in[0, T])
$$

associated with $T,\left(\alpha_{n}\right),\left(\beta_{n}\right)$, and $\left(\lambda_{n}\right)$ satisfies the uniqueness property

$$
\forall t \in[0, T] \quad f(t)=0 \quad \Rightarrow \quad \forall n \in \mathbb{N} \quad \alpha_{n}=\beta_{n}=0
$$

Proof. For simplicity we assume that $\lambda_{n}>0$ for all $n \in \mathbb{N}$. (Since the sequence $\left(\lambda_{n}\right)$ is increasing and satisfies $\lambda_{n} \geq(n-1) \gamma+c \lambda_{1}$ for large enough $n$ by the gap condition (38), only a finite number of $\lambda_{n}$ 's are negative.

\footnotetext{
7 The "fixed-profile" form of the control also appears in optics applications when the holding beam has a fixed shape.
} 
The proof is very similar in this case.) The proof is based on the following inequalities due to Hilbert

$$
\begin{array}{r}
\sum_{j, k=0}^{\infty} \frac{\left|a_{j} b_{k}\right|}{j+k+1} \leq \pi\|a\|_{\ell^{2}}\|b\|_{\ell^{2}} \\
\left|\sum_{j \neq k}^{\infty} \frac{\left|a_{j} b_{k}\right|}{j-k}\right| \leq \pi\|a\|_{\ell^{2}}\|b\|_{\ell^{2}}
\end{array}
$$

where $\left(a_{k}\right)_{k=0,1, \ldots,}\left(b_{k}\right)_{k=0,1, \ldots} \in \ell^{2} ; c f . \quad[16]$. It is also known that $\pi$ is the optimal constant in these inequalities. Since $f(t) \equiv 0$ in $[0, T]$, we have $\int_{0}^{T} f^{2}(t) \mathrm{d} t=0$. This, together with $(40)$, implies

$$
\begin{aligned}
\sum_{n=1}^{\infty} \alpha_{n}^{2}\left\{\frac{T}{2}-\frac{\sin \left(2 \lambda_{n} T\right)}{4 \lambda_{n}}\right\}+\sum_{n=1}^{\infty} \beta_{n}^{2}\left\{\frac{T}{2}+\frac{\sin \left(2 \lambda_{n} T\right)}{4 \lambda_{n}}\right\}= & \sum_{j \neq k} \alpha_{j} \alpha_{k}\left\{\frac{\sin \left(\left(\lambda_{j}+\lambda_{k}\right) T\right)}{2\left(\lambda_{j}+\lambda_{k}\right)}-\frac{\sin \left(\left(\lambda_{j}-\lambda_{k}\right) T\right)}{2\left(\lambda_{j}-\lambda_{k}\right)}\right\} \\
& -\sum_{j \neq k} \beta_{j} \beta_{k}\left\{\frac{\sin \left(\left(\lambda_{j}+\lambda_{k}\right) T\right)}{2\left(\lambda_{j}+\lambda_{k}\right)}+\frac{\sin \left(\left(\lambda_{j}-\lambda_{k}\right) T\right)}{2\left(\lambda_{j}-\lambda_{k}\right)}\right\} \\
& +2 \sum_{j \neq k} \alpha_{j} \beta_{k}\left\{\frac{1}{2\left(\lambda_{j}+\lambda_{k}\right)}\left[1-\cos \left(\left(\lambda_{j}+\lambda_{k}\right) T\right)\right]\right. \\
& \left.+\frac{1}{2\left(\lambda_{j}-\lambda_{k}\right)}\left[1-\cos \left(\left(\lambda_{j}-\lambda_{k}\right) T\right)\right]\right\} \\
& +2 \sum_{n=1}^{\infty} \alpha_{n} \beta_{n} \frac{1}{4 \lambda_{n}}\left[1-\cos \left(2 \lambda_{n} T\right)\right] .
\end{aligned}
$$

Set $\alpha:=\left(\sum_{j=1}^{\infty} \alpha_{j}^{2}\right)^{1 / 2}$ and $\beta:=\left(\sum_{j=1}^{\infty} \beta_{j}^{2}\right)^{1 / 2}$. Then

$$
\begin{aligned}
\frac{T}{2}\left(\alpha^{2}+\beta^{2}\right) \leq & \frac{1}{2 \lambda_{1}}\left(\alpha^{2}+\beta^{2}+2 \alpha \cdot \beta\right)+\frac{1}{2}\left(\sum_{j \neq k} \frac{\left|\alpha_{j} \alpha_{k}\right|}{\lambda_{j}+\lambda_{k}}+\sum_{j \neq k} \frac{\left|\alpha_{j} \alpha_{k}\right|}{\left|\lambda_{j}-\lambda_{k}\right|}+\sum_{j \neq k} \frac{\left|\beta_{j} \beta_{k}\right|}{\lambda_{j}+\lambda_{k}}+\sum_{j \neq k} \frac{\left|\beta_{j} \beta_{k}\right|}{\left|\lambda_{j}-\lambda_{k}\right|}\right) \\
& +2\left(\sum_{j \neq k} \frac{\left|\alpha_{j} \beta_{k}\right|}{\lambda_{j}+\lambda_{k}}+\sum_{j \neq k} \frac{\left|\alpha_{j} \beta_{k}\right|}{\left|\lambda_{j}-\lambda_{k}\right|}\right) .
\end{aligned}
$$

Thanks to (38) we have $\left|\lambda_{j}-\lambda_{k}\right| \geq \gamma|j-k|$ and $\lambda_{j}+\lambda_{k} \geq \gamma\left(j+k+1-3+\frac{\lambda_{1}}{\gamma}\right)$. So, assuming that $-3+\frac{\lambda_{1}}{\gamma} \geq 0$ (this condition can always be satisfied by decreasing $\gamma$ if necessary) and using (41) and (42), we get

$$
\alpha^{2}+\beta^{2} \leq \frac{2}{T}\left(\frac{1}{\lambda_{1}}+\frac{\pi}{\gamma}\right)\left(\alpha^{2}+\beta^{2}\right)+\frac{4 \pi}{T \gamma} \alpha \cdot \beta \leq \frac{2}{T}\left(\frac{1}{\lambda_{1}}+\frac{3 \pi}{\gamma}\right)\left(\alpha^{2}+\beta^{2}\right)
$$

Under the condition

the inequality (43) implies

$$
\frac{2}{T}\left(\frac{1}{\lambda_{1}}+\frac{3 \pi}{\gamma}\right)<1 \quad \Longleftrightarrow \quad T>T_{0}:=\frac{1}{\lambda_{1}}+\frac{3 \pi}{\gamma}
$$

This concludes the proof.

$$
\alpha=\beta=0 \quad \Longleftrightarrow \quad \alpha_{n}=\beta_{n}=0 \quad(\forall n \in \mathbb{N})
$$


Theorem 7. Assume that the operator A possesses an orthonormal basis $\left(\varphi_{n}\right)_{n \in \mathbb{N}}$ of eigenfunctions in $L^{2}(\Omega)$ and denote by $\left(\lambda_{n}\right)_{n \in \mathbb{N}}$ the corresponding eigenvalues. Let $h \in L^{2}(\Omega)$ be a non-trivial function. Then

(i) If equation (36) is approximately controllable, then

$$
\left(h, \varphi_{n}\right)_{L^{2}(\Omega)} \neq 0 \quad \text { for all } n \in \mathbb{N}
$$

(ii) If (44) holds and, in addition, the sequence $\left(\lambda_{n}\right)_{n \in \mathbb{N}}$ satisfies the gap condition (38), then equation (36) is approximately controllable in the complex sense, provided that the control time satisfies the condition $T>2 \pi / \gamma$.

(iii) If (44) holds and the sequence $\left(\lambda_{n}\right)_{n \in \mathbb{N}}$ satisfies the gap condition (38) and, in addition, the eigenvalues $\lambda_{n}$ are all non-zero, then equation (36) is approximately controllable in the real sense provided that the control time satisfies the condition $T \geq T_{0}$, where $T_{0}$ is the constant appearing in the assertion of the previous lemma.

Proof. (i) Referring to the nomenclature of Section 1, the operator $B: \mathbb{K} \rightarrow L^{2}(\Omega)$ is defined by

$$
B g:=-i g \cdot h
$$

where $\mathbb{K}=\mathbb{C}$ (complex case) or $\mathbb{K}=\mathbb{R}$ (real case). The adjoint operator $B^{*}: L(\Omega) \rightarrow \mathbb{K}$ is given by

$$
B^{*} w= \begin{cases}i(w, h)_{L^{2}(\Omega)}, & \text { complex case } \\ -\Im(w, h)_{L^{2}(\Omega)}, & \text { real case. }\end{cases}
$$

The approximate controllability of (36) is equivalent to the following property:

$$
\forall t \in[0, T] \quad(S(-t) v, h)_{L^{2}(\Omega)}=0 \quad \Rightarrow \quad v \equiv 0,
$$

where $S$ denotes the Schrödinger group which may be written as

$$
S(t) v=\sum_{n=1}^{\infty} \mathrm{e}^{-i \lambda_{n} t}\left(v, \varphi_{n}\right)_{L^{2}(\Omega)} \varphi_{n} .
$$

So if $\left(h, \varphi_{m}\right)_{L^{2}(\Omega)}$ was equal to zero for some index $m \in \mathbb{N}$, we would get

$$
\left(S(-t) \varphi_{m}, h\right)_{L^{2}(\Omega)}=\mathrm{e}^{i \lambda_{m} t}\left(\varphi_{m}, h\right)_{L^{2}(\Omega)}=0 \quad(\forall t \in[0, T]),
$$

which is impossible in view of property $(47)$, as $v=\varphi_{m} \neq 0$.

In the real case the approximate controllability of (36) is equivalent to the property

$$
\forall t \in[0, T] \quad \Im(S(-t) v, h)_{L^{2}(\Omega)}=0 \quad \Rightarrow \quad v \equiv 0,
$$

and the assumption $\left(\varphi_{m}, h\right)_{L^{2}(\Omega)}=0$ will again lead to a contradiction.

(ii) We want to show that condition (47) is satisfied. To this end, we assume that $\left(S(-t) u_{0}, h\right)_{L^{2}(\Omega)}=0$ for all $\overline{t \in}[0, T]$. Then the function $\psi:[0, T] \rightarrow \mathbb{C}$, defined by

$$
\psi(t)=\left(S(-t) u_{0}, h\right)=\sum_{n=1}^{\infty} \mathrm{e}^{i \lambda_{n} t}\left(u_{0}, \varphi_{n}\right)\left(\varphi_{n}, h\right)=: \sum_{n=1}^{\infty} \mathrm{e}^{i \lambda_{n} t} c_{n}
$$

$\left(c_{n}:=\left(u_{0}, \varphi_{n}\right)\left(\varphi_{n}, h\right)\right)$, is identically zero in $[0, T]$. Note that $\left(c_{n}\right) \in \ell^{1} \cap \ell^{2}$, since $\left(u_{0}, \varphi_{n}\right),\left(\varphi_{n}, h\right) \in \ell^{2}$ and $\left|\left(u_{0}, \varphi_{n}\right)\right| \leq\left\|u_{0}\right\|_{L^{2}(\Omega)}$; in particular, we have $\psi \in C[0, T]$. Now, if $T>2 \pi / \gamma$, applying Lemma 3.1 results in $c_{n}=0$ for all $n \in \mathbb{N}$ which in turn implies $u_{0} \equiv 0$, as $\left(\varphi_{n}, h\right) \neq 0$ by $(44)$. 
(iii) The proof is similar to the proof of (ii): express $f(t):=\Im \psi(t)$ as

$$
f(t)=\sum_{n=1}^{\infty}\left\{\alpha_{n} \sin \left(\lambda_{n} t\right)+\beta_{n} \cos \left(\lambda_{n} t\right)\right\} \quad(t \in[0, T])
$$

Assuming $f \equiv 0$ and utilizing Lemma 3.2 will again imply $u_{0} \equiv 0$. This concludes the proof of the theorem.

\subsection{Additive control for NLS}

We consider the exact distributed control problem for the (free) nonlinear Schrödinger equation

$$
\left\{\begin{array}{l}
i u_{t}=-u_{x x}-\alpha|u|^{2} u+\chi_{\omega}(x) \cdot h(t, x), \quad x \in \Omega \subset \mathbb{R}, \quad t \in[0, T] \\
u(0, x)=u_{0}(x), \quad u(T, x)=u_{1}(x)
\end{array}\right.
$$

which was presented in [20]. Here $\alpha \in \mathbb{R}$ is a fixed parameter, $\Omega \subset \mathbb{R}$ is an open interval and $\chi_{\omega}$ denotes the characteristic function of an open and and bounded subinterval $\omega \subset \Omega$ of $\Omega$. We consider equation (49) with periodic boundary conditions on the interval $\Omega=[0,1]$, i.e. we work in the function space $H_{p e r}^{1}(0,1)$.

Theorem 8. Let $T>0, \omega=(a, b) \subset[0,1]$ an open interval. Then there exists a constant $C=C(T, \omega)>0$ such that for all $u_{0}, u_{1} \in H_{\text {per }}^{1}(0,1)$ with

$$
\left\|u_{0}\right\|_{H^{1}}+\left\|u_{1}\right\|_{H_{1}} \leq C
$$

there exists a control function $h \in C\left([0,1], H_{\text {per }}^{1}(0,1)\right)$ and a control state $u \in C\left([0,1], H_{\text {per }}^{1}(0,1)\right)$ which is a $H^{1}$-solution of (49). Furthermore there exists a constant $\tilde{C}=\tilde{C}(T, \omega)$ such that

$$
\sup _{t \in[0, T]}\|h(t, .)\|_{H^{1}} \leq \tilde{C}\left[\left\|u_{0}\right\|_{H^{1}}+\left\|u_{1}\right\|_{H^{1}}\right] .
$$

\section{Remark 8.}

(i) A proof for the theorem was given in [20]. The proof involves the following steps: linearizing equation (49) by freezing the nonlinearity; showing that the linearized problem is exactly controllable by applying the Hilbert Uniqueness Method due to Lions; and employing Schauder's Fixed Point Theorem to solve the nonlinear problem.

(ii) A generalized version of the theorem states that equation (49) is locally approximately controllable in the vicinity of stationary solutions. The details of this result and its proof will appear elsewhere.

Acknowledgements. This research was supported by operating grants from the Natural Sciences and Engineering Research Council of Canada, and by research funds from the German Research Foundation (DFG). HL \& HT would like to acknowledge the hospitality of the Department of Mathematics and Statistics at the University of Victoria, where most of this research was done. The authors would also like to thank PIMS for providing an outstanding research environment at BIRS. This paper was finished during a PIMS-sponsored "Focussed Research Group" event at BIRS in Aug./Sep. 2004. Finally, the authors would also like to thank the referees for their thoughtful remarks, which helped improve the paper significantly.

\section{REFERENCES}

[1] F.Kh. Abdullaev and J. Garnier, Collective oscillations of one-dimensional Bose-Einstein gas under varying in time trap potential and atomic scattering length. Phys. Rev. A 70 (2004) 053604.

[2] G. Bachman and N. Narici, Functional Analysis. Academic Press, N.Y. (1966).

[3] J. Ball, J. Marsden and M. Slemrod, Controllability for distributed bilinear systems. SIAM J. Contr. Opt. 20 (1982) 575-597. 
[4] C. Bardos, G. Lebeau and J. Rauch, Sharp sufficient conditions for the observation, control, and stabilization of waves from the boundary. SIAM J. Contr. Opt. 30 (1992) 1024-1065.

[5] L. Baudouin, A bilinear optimal control problem applied to a time dependent Hartree-Fock equation coupled with classical nuclear dynamics. Portugaliae Mat. (To appear).

[6] L. Baudouin, Existence and regularity of the solution of a time dependent Hartree-Fock equation coupled with a classical nuclear dynamics. Rev. Mat. Complut. 18 (2005) 285-314.

[7] L. Baudouin and J.-P. Puel, Bilinear optimal control problem on a Schrödinger equation with singular potentials. Preprint (2004).

[8] K. Beauchard, Local controllability of a 1-D Schrödinger equation, J. Math. Pures Appl. 84 (2005) 851-956.

[9] K. Beauchard and J.M. Coron, Controllability of a quantum particle in a moving potential well. J. Funct. Anal. 232 (2006) 328-389.

[10] P.W. Brumer and M. Shapiro, Principles of the Quantum Control of Molecular Processes. Wiley-VCH, Berlin (2003).

[11] R. Carles, Linear vs. nonlinear effects for nonlinear Schrödinger equations with potential. Commun. Contemp. Math. 7(4) (2005) 483-508.

[12] E. Cancès and C. LeBris, On the time-dependent Hartree-Fock equations coupled with classical nuclear dynamics. Math. Mod. Meth. Appl. Sci. 9 (1999) 963-990.

[13] E. Cancès, C. LeBris and M. Pilot, Contrôle optimale bilinéaire d'une équation de Schrödinger. C. R. Acad. Sci. Paris, Sér. $1330(2000) 567-571$.

[14] J.W. Clark, D.G. Lucarelli and T.J. Tarn, Control of quantum systems. Int. J. Mod. Phys. B 17 (2003) 5397-5412.

[15] C. Fabre, Résultats de contrôlabilité exacte interne pour l'équation de Schrödinger at leurs limites asymptotiques, Application à certaines équations de plaques vibrantes. Asymptotic Analysis 5 (1992) 343-379.

[16] H. Helson, Harmonic Analysis. Addison-Wesley, Reading (1983).

[17] M. Holthaus and S. Stenholm, Coherent control of self-trapping transition. Eur. Phys. J. B 20 (2001) 451-467.

[18] G.M Huang, Tarn T.J and J.W. Clark, On the controllability of quantum-mechanical systems. J. Math. Phys. 24 (1983) $2608-2618$.

[19] H. Husimi, Miscellanea in elementary quantum mechanics II. Prog. Theor. Phys. 9 (1953) 381-402.

[20] R. Illner, H. Lange and H. Teismann, A note on the exact internal control of nonlinear Schrödinger equations. CRM Proc. Lecture Notes 33 (2003) 127-137.

[21] A.E. Ingham, Some trigonometric inequalities with applications to the theory of series. Math. Z. 41 (1936) 367.

[22] J.L. Journé, A. Soffer and C.D. Sogge, Decay estimates for Schrödinger operators. Commun. Pure Appl. Math. 44 (1991) $573-604$.

[23] K.H. Kerner, Note on the forced and damped oscillator in quantum mechanics. Can. J. Phys. 36 (1958) 371-377.

[24] C. Lan, T.J. Tarn, Q.-S. Chi and J.W. Clark, Analytic controllability of time-dependent quantum control systems. J. Math. Phys. 46 (2005) 052102

[25] I. Lasiecka and R. Triggiani, Optimal regularity, exact controllability and uniform stabilization of Schrödinger equations with Dirichlet controls. Differ. Int. Equ. 5 (1992) 571-535.

[26] I. Lasiecka and R. Triggiani, Control theory for partial differential equations, continuous and approximation theories. I 83 II. Cambridge University Press, Cambridge (2000).

[27] I. Lasiecka, R. Triggiani and X. Zhang, Global uniqueness, observability and stabilization of nonconservative Schrödinger equations via pointwise Carleman estimates. I. $H^{1}(\Omega)$-estimates. J. Inverse Ill-Posed Probl. 12 (2004) 43-123.

[28] I. Lasiecka, R. Triggiani and X. Zhang, Global uniqueness, observability and stabilization of nonconservative Schrödinger equations via pointwise Carleman estimates. II. $L_{2}(\Omega)$-estimates. J. Inverse Ill-Posed Probl. 12 (2004) 183-231.

[29] G. Lebeau, Contrôle de l'équation de Schrödinger. Jour. Math. Pures Appl. 71 (1992) 267-291.

[30] C. LeBris, Control theory applied to quantum chemistry, some tracks, in Conf. Int. contrôle des systèmes gouvernés par des équations aux derivées partielles. ESAIM Proc. 8 (2000) 77-94.

[31] C. LeBris, Computational Chemistry, in Handbook of Numerical Analysis, C. LeBris, Ph.G. Ciarlet Eds. North-Holland, Amsterdam (2003).

[32] J.-L. Lions, Contrôlabilité exacte, perturbations et stabilisation de systèmes distribués. Tome 1 \& 2. Masson, Paris (1988).

[33] E. Machtyngier, Exact controllability for the Schrödinger equation. SIAM J. Contr. Opt. 32 (1994) 24-34.

[34] E. Machtyngier and E. Zuazua, Stabilization of the Schrödinger equation. Portugaliae Mat. 51 (1994) 243-256.

[35] M. Mirrahimi and P. Rouchon, Controllability of quantum harmonic oscillators. IEEE Trans. Automatic Control 49 (2004) 745-747.

[36] K.-D. Phung, Observability and control of Schrödinger equations. SIAM J. Contr. Opt. 40 (2001) 211-230.

[37] S.A. Rice and M. Zhao, Optical Control of Molecular Dynamics. John Wiley \& Sons, New York (2000).

[38] D.L. Russell, Controllability and stabilizability theory for linear partial differential equations, recent progress and open questions. SIAM Rev. (1978) 20 639-739.

[39] S.G. Schirmer, J.V. Leahy and A.I. Solomon, Degrees of controllability for quantum systems and application to atomic systems. J. Phys. A 35 (2002) 4125-4141.

[40] A.P. Shustov, Coherent states and energy spectrum of the anharmonic osciallator. J. Phys. A 11 (1978) 1771-1780. 
[41] E.M. Stein, Singular Integrals and Differentiability Properties of Functions. Princeton University Press (1974).

[42] G. Turinici, Analyse de méthodes numériques de simulation et contrôle en chimie quantique. Ph.D. Thesis, Univ. Paris VI (2000).

[43] G. Turinici, Controllable quantities for bilinear quantum systems, in Proc. of the 39th IEEE Conference on Decision and Control, Sydney, Australia (2000) 1364-1369.

[44] R.M. Young, An Introduction to Nonharmonic Fourier Series. Academic Press, New York (1980).

[45] J. Zabczyk, Introduction to Control Theory. Birkhäuser, Basel (1994).

[46] E. Zuazua, Remarks on the controllability of the Schrödinger equation. CRM Proc. Lecture Notes 33 (2003) 193-211. 